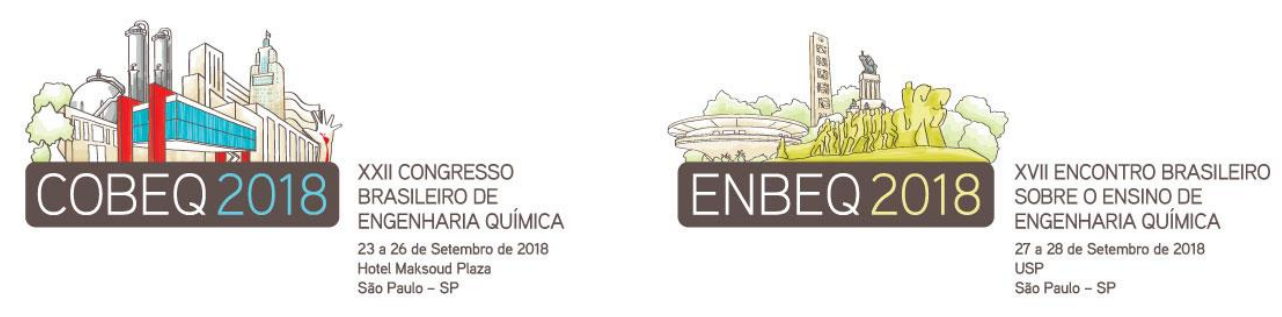

\title{
VISCOSIDADE APARENTE DA POLPA DE MANGA ESPADA
}

\author{
LOPES, $\mathrm{CCB}^{1}$, OLIVEIRA, RGM ${ }^{2}, \mathrm{MELO}, \mathrm{JCS}^{3} \mathrm{COSTA} \mathrm{CHC}^{4}, \mathrm{BADARO}, \mathrm{ADS}^{5}$, \\ OLIVEIRA, $\mathrm{KP}^{6}$ \\ 1,2,3,4 Instituto Federal do Rio Grande do Norte (Campus Caicó) \\ ${ }^{5}$ Universidade Federal do Rio Grande do Norte, Departamento de Engenharia Têxtil \\ ${ }^{6}$ Instituto Federal do Rio Grande do Norte (Campus Cidade Alta) \\ E-mail para contato: carlos.soares@ifrn.edu.br
}

\begin{abstract}
RESUMO - A viscosidade aparente da polpa de fruta, por exemplo, varia com a temperatura e concentração a ponto de inviabilizar o seu processamento. Logo, o objetivo deste trabalho é estudar o comportamento da viscosidade aparente da polpa de manga espada nas temperaturas de $15,25,35,45$ e $55^{\circ} \mathrm{C}$ e na concentração de $20^{\circ} \mathrm{Brix}$. As medidas das viscosidades aparentes da polpa de manga espada foram determinadas utilizando um viscosímetro rotativo analógico da marca QUIMIS, em diferentes velocidades de rotação $(0,6,1,5,3,0,6,0,12,30$ e $60 \mathrm{rpm})$, nas temperaturas de 15, 25, 35,45 e $55^{\circ} \mathrm{C}$. Os valores das viscosidades aparentes da polpa de manga espada, na concentração de $20^{\circ}$ Brix, foram influenciados pelas velocidades de rotação e as temperaturas estudadas, cujas viscosidades aparentes diminuíram com o aumento dessas variáveis. A polpa de manga espada apresentou comportamento de um fluido nãonewtoniano do tipo pseudoplástico. As equações ajustadas aos dados experimentais das viscosidades aparentes da polpa de manga apresentaram coeficiente de determinação $R^{2}>0,98$.
\end{abstract}

\section{INTRODUÇÃO}

A manga é uma fruta de regiões tropicais e subtropicais, que é procurado (in natura) pelas indústrias para fazer seus derivados: néctares, sucos, sorvetes e sobremesas. Durante o processamento de sucos e polpas de frutas, frequentemente utilizam-se tratamentos térmicos, os quais são influenciados por inúmeros fatores, como a quantidade de sólidos solúveis e insolúveis, a distribuição do tamanho de partículas, temperatura, pressão, fazendo com que o estudo da influência desses fatores sobre o comportamento da viscosidade aparente desses produtos seja de grande importância (Miranda et al., 2012; Braga et al.; 2013).

A viscosidade aparente das polpas de frutas depende de suas características físicoquímicas e estrutura e pode ser utilizada como parâmetro de qualidade e correlacionada com a textura e análise sensorial (Feitosa et al., 2015).

Muitos fluidos alimentícios, principalmente polpas e sucos de frutas, são classificados como fluidos não-newtonianos do tipo pseudoplástico, como por exemplo, a polpa de murta, polpa de noni, graviola e manga centrifugada (Feitosa et al., 2015; Sousa et al., 2017; Miranda et al., 2012; Vidal et al., 2006). 


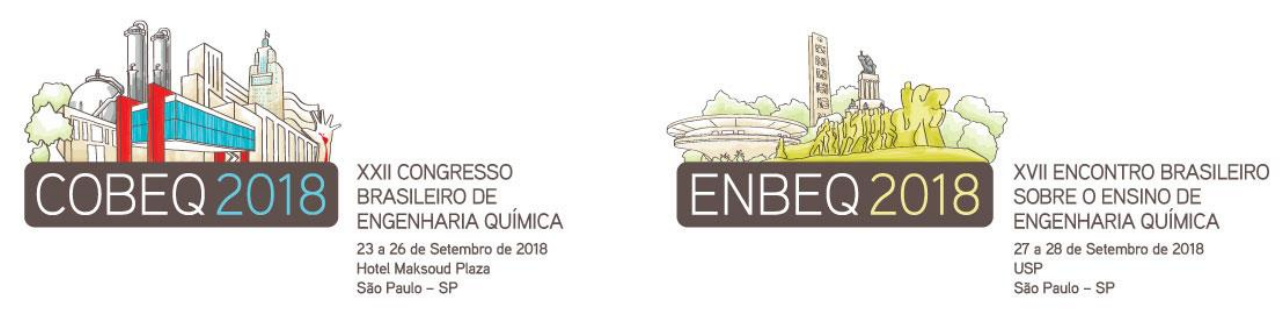

O objetivo deste trabalho é determinar experimentalmente a viscosidade aparente da polpa de manga espada nas temperaturas de $15,25,35,45$ e $55^{\circ} \mathrm{C}$, na concentração de $20^{\circ}$ Brix, a fim de avaliar a influência da temperatura na viscosidade aparente na polpa da manga espada.

\section{MATERIAIS E MÉTODOS}

As mangas foram adquiridas no comércio da cidade de Caicó - RN, sendo escolhidas as maduras e com uma textura firme, sem doenças nem bolores. Posteriormente, foram lavadas em água corrente e depois imersas em uma solução de hipoclorito de sódio de 50 ppm, durante 15 minutos; em seguida, foram enxaguadas e despolpadas.

Com um viscosímetro rotativo analógico da marca QUIMIS, modelo Q860A24, foram realizadas leituras de torque nas velocidades de rotação $(0,6,1,5,3,0 ; 6,0 ; 12 ; 30$ e 60 rpm), nas temperaturas de $15,25,35,45$ e $55^{\circ} \mathrm{C}$, realizados em triplicatas. Após as leituras de torque no viscosímetro, essas foram multiplicadas por um fator $\mathrm{F}$ em função do spindle utilizado (de acordo com o manual do equipamento), obtendo a viscosidade aparente (Equação 1).

$y=F . \alpha i$

Onde: $\mathrm{y}$ - viscosidade aparente (Pa.s), F - fator (admensional), $\alpha \mathrm{i}$ - torque

Foi aplicado o modelo matemático (Equação 2) para verificar a dependência da viscosidade aparente (Pa.s) da polpa da manga espada em relação a velocidade de rotação (rpm).

$y=a \cdot x^{b}$

Onde: $\mathrm{y}$ - viscosidade aparente (Pa s); $\mathrm{x}$ - velocidade de rotação; $a$ e b - constantes da equação.

Os parâmetros do modelo foram determinados utilizando-se o software STATISTICA, versão 5.0, através de regressão não linear, utilizando o método QuaseNewton. Já os valores das viscosidades aparentes da polpa de manga espada foram avaliados por meio de análises de Tukey através do programa ASSISTAT versão 7.7 Beta (Silva e Azevedo, 2009).

\section{RESULTADO E DISCUSSÕES}

Na Tabela 1, têm-se os valores experimentais das viscosidades aparentes ( $\mathrm{Pa}$ s) da polpa de manga espada na concentração de $20^{\circ}$ Brix, em função das velocidades de rotação, nas temperaturas de $15,25,35,45$ e $55^{\circ} \mathrm{C}$. Observa-se na Tabela 1 que o aumento da velocidade de rotação ocasionou uma tendência de diminuição dos valores médios de viscosidade aparente da polpa de manga espada em todas as temperaturas estudadas. No entanto, nas velocidades de 30 e $60 \mathrm{rpm}$, os valores de viscosidade tenderam permanecer constante, de acordo com o teste de Tukey a $5 \%$ de probabilidade. Mesmo comportamento observado por Lima et al. (2014) a viscosidade aparente da polpa de abacaxi.

Verificam-se ainda que os valores das viscosidades aparentes da polpa de manga espada tenderam a diminuir com o aumento da temperatura, para a mesma velocidade de rotação, embora na velocidade de $60 \mathrm{rpm}$, os valores das viscosidades aparentes não apresentaram diferença 

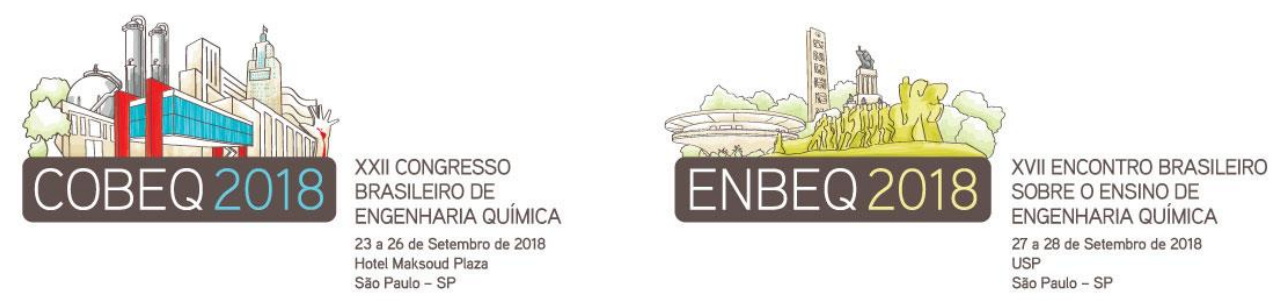

estatística. Miranda et al., (2012), verificaram que a viscosidade aparente da polpa de graviola, nas concentrações de 12,17 e $25^{\circ}$ Brix, diminuíram com o aumento da temperatura de 10 a $50{ }^{\circ} \mathrm{C}$.

Tabela 1 - Viscosidade aparente (Pa.s) da polpa de manga com $20^{\circ}$ Brix em função da velocidade de rotação e temperatura

\begin{tabular}{cccccc}
\hline Velocidade de & \multicolumn{5}{c}{ Temperatura $\left({ }^{\circ} \mathrm{C}\right)$} \\
\cline { 2 - 6 } rotação & 15 & 25 & 35 & 45 & 55 \\
\hline 0,6 & $146,67 \mathrm{aA}$ & $131,67 \mathrm{aB}$ & $116,67 \mathrm{aC}$ & $105,00 \mathrm{aD}$ & $68,33 \mathrm{aE}$ \\
1,5 & $104,67 \mathrm{bA}$ & $80,00 \mathrm{bB}$ & $60,00 \mathrm{bC}$ & $55,33 \mathrm{bD}$ & $29,33 \mathrm{bE}$ \\
3 & $56,33 \mathrm{cA}$ & $49,67 \mathrm{cB}$ & $35,00 \mathrm{cC}$ & $33,33 \mathrm{cC}$ & $19,33 \mathrm{cD}$ \\
6 & $31,67 \mathrm{dA}$ & $29,33 \mathrm{dA}$ & $21,00 \mathrm{~dB}$ & $19,83 \mathrm{~dB}$ & $15,00 \mathrm{dC}$ \\
12 & $17,83 \mathrm{eA}$ & $17,50 \mathrm{eA}$ & $12,67 \mathrm{eB}$ & $12,33 \mathrm{eBC}$ & $9,08 \mathrm{eC}$ \\
30 & $8,57 \mathrm{fA}$ & $8,60 \mathrm{fA}$ & $6,17 \mathrm{fAB}$ & $6,37 \mathrm{fAB}$ & $4,23 \mathrm{fB}$ \\
60 & $5,30 \mathrm{fA}$ & $5,17 \mathrm{fA}$ & $3,63 \mathrm{fA}$ & $3,85 \mathrm{fA}$ & $2,77 \mathrm{fA}$ \\
\hline
\end{tabular}

DMS colunas $=3,65 ;$ DMS para linhas $=3,37 ; \mathrm{MG}=38,64 \mathrm{~Pa} \mathrm{~s} ; \mathrm{CV}=3,81 \%$

MG: Média geral; CV: Coeficiente de variação e DMS: Desvio mínimo significativo.

Obs.: Médias seguidas das mesmas letras minúsculas nas colunas e maiúsculas nas linhas não diferem estatisticamente pelo teste de Tukey, a 5\% de probabilidade

Segundo Vidal et a1., (2006), esse comportamento deve-se em razão do aumento da temperatura diminuir a viscosidade aparente, aumentando a mobilidade das partículas em suspensão, diminuindo consequentemente a viscosidade da polpa de fruta.

Observa-se na Figura 1 que a polpa da manga espada apresentou comportamento de um fluido não-newtoniano do tipo pseudoplástico, pois ocorreu a diminuição da viscosidade aparente da polpa de manga com o aumento da velocidade de rotação.

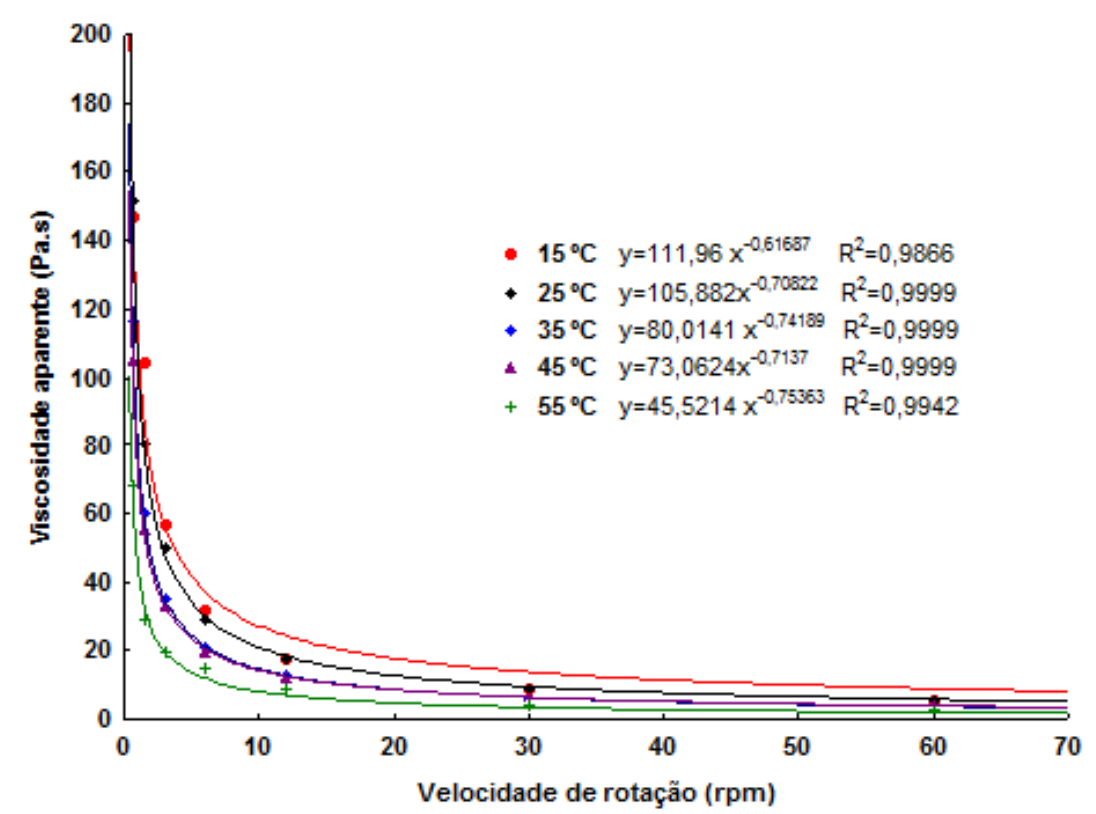

Figura 1 - Viscosidade aparente em função da velocidade de rotação da polpa de manga espada nas temperaturas de $15,25,35,45$ e $55^{\circ} \mathrm{C}$. 


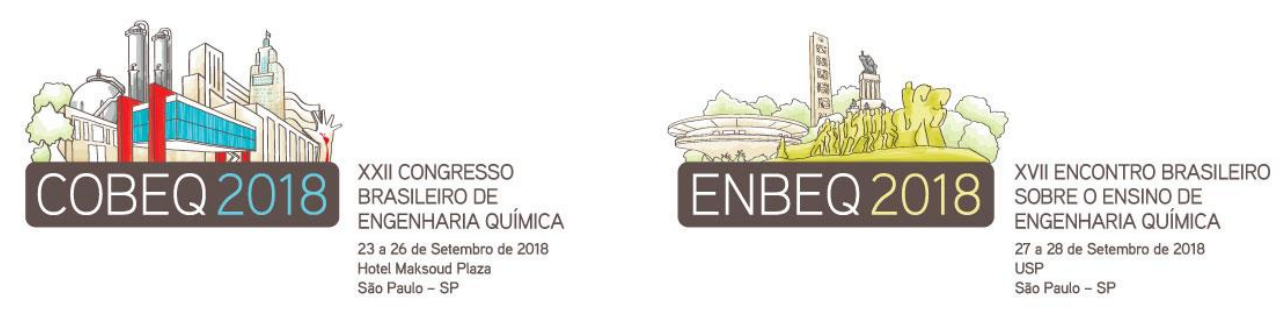

Sousa et al. (2017) estudando o comportamento reológico da polpa de noni (Morinda citrifolia L.) integral e concentradas a 30 e $50^{\circ}$ Brix, observaram que a polpa de noni era fluido não newtonianos com comportamento pseudoplástico.

Na Figura 1, observam-se ainda, que as equações ajustadas aos dados experimentais, das viscosidades aparentes da polpa de manga espada, podem ser estimadas em relação a velocidade de rotação, pois o menor valor do coeficiente de determinação encontrado foi superior a 0,98. Miranda et al. (2012) verificaram que os coeficientes de determinação $\left(\mathrm{R}^{2}\right)$ foram superiores a 0,98 , indicando um bom ajuste da equação aplicada aos dados experimentais.

\section{CONCLUSÃO}

Os valores das viscosidades aparentes da polpa de manga espada foram influenciados pelas velocidades de rotação e pelas temperaturas estudadas, cujas viscosidades aparentes diminuíram com o aumento dessas variáveis. A polpa de manga espada apresentou comportamento de um fluido não- newtoniano do tipo pseudoplástico. As equações ajustadas aos dados experimentais das viscosidades aparentes da polpa de manga espada podem ser usadas para estimar a viscosidade aparente da polpa de manga espada, pois $\mathrm{R}^{2}>0,98$.

\section{REFERÊNCIAS}

BRAGA ACC, RODRIGUES AMC, SILVA LHM, ARAÚJO LA. Avaliação da influência da temperatura e do tratamento enzimático no comportamento reológico do suco de abacaxi pérola (Ananas Comosus L. merr). Rev. Bras. Frutic., v.35, p.226-237. 2013.

FEITOSA RM, FIGUEIRÊDO RMF, QUEIROZ AJM, SOUZA EP, SILVA VM. Viscosidade aparente da polpa de murta integral em diferentes temperaturas. Rev. Caatinga, v.28, p. 235-243, 2015.

LIMA FCS, MELO JCS, PEREIRA ED, COSTA CHC, MIRANDA VAM. Estudo da viscosidade aparente da polpa de abacaxi integral. CBQ $54^{\circ}$ Congresso Brasileiro De Química, Natal/Rio grande do Norte, de 03/11 a 07/11 de 2014.

MIRANDA VAM, QUEIROZ AJM, FIGUEIRÊDO RMF, SANTOS DC. Viscosidade aparente de polpas de graviola com diferentes concentrações. Rev. Bras. Prod. Agroind., v.13, p.363-374, 2012.

SILVA FAS, AZEVEDO CAV. Principal components analysis in the software assistat-statistical attendance. In: WORLD CONGRESS ON COMPUTERS IN AGRICULTURE, 7, Reno-NVUSA: Americam Society of Agricultural and Biological Engineers, 2009.

SOUSA SF, QUEIROZ AJM, FIGUEIRÊDO RMF, SILVA FB. Comportamento reológico das polpas de noni integral e concentradas. Braz. J. Food Technol., v. 20, e2016067, 2017.

VIDAL JRMB, SIERAKOWSKI MR, HAMINIUK CWI, MASSON, ML. Rheological properties of centrifuged mango (Mangifera indica L. cv. Keitt) pulp. Ciênc. Agrotec. v. 30, p. 955-960, 2006. 